\title{
Various angle periods of parabolic coincidence fringes in violation of Bell inequality with high-dimensional two-photon entanglement
}

\author{
Hsiao-Chih Huang ${ }^{1,2, *}$ \\ ${ }^{1}$ Institute of Atomic and Molecular Sciences, Academia Sinica, Taipei 106, Taiwan \\ ${ }^{2}$ HC Photonics Corporation, No. 2, Technology Rd. V, Hsinchu 300, Taiwan
}

*Email: d93222016@ntu.edu.tw

\begin{abstract}
Two quantum states of two half-charge optical vortexes with relative azimuthal angle $\pi$ are orthogonal, by which two half-charge spiral phase plates with intersection angle $\pi$ can be used to demonstrate a parabolic coincident fringe in the Bell inequality experiment with high-dimensional two-photon orbital angular momentum entanglement and to thereby obtain a strong Bell parameter of $3 \frac{1}{5}$. I theoretically demonstrate various orthogonal relations between two quantum states, each of which is a state with a rotational symmetry superposition made up of $n$ fractional orbital angular momentum states, where $n \in N$. I propose a Bell inequality experiment with two $n$-section spiral phase plates that have these two quantum states, whereby various angle periods with parabolic coincident fringes $2 \pi / n$ and Bell's parameter $3 \frac{1}{5}$ in each period can be obtained.
\end{abstract}




\section{INTRODUCTION}

Over the past few decades, entangled photon pairs obtained by spontaneously parametric down-conversion (SPDC) [1, 2] have been used to experimentally verify the quantum nonlocality in the violation of the Bell inequality [3]. For two-particle and two-dimensional entanglement, the measured coincidence fringe of the pairs is the square of the sinusoid and Bell's parameter is $2 \sqrt{2}$ [4], as proved using photon polarization [5], phase in spatial parity of one-dimensional transverse field (Hermite-Gaussian 10 mode) [6], phase with ghost imaging [7], and orbital angular momentum (OAM) of identical topological charge [8, 9]. In the first three cases, the angle periods of the coincidence fringes are respectively fixed at $\pi, 2 \pi$, rand $\pi$. However, in the last case, the angle periods vary over the range $\pi / m$, where $m$ is the topological charge. In this case, the period variable is attributed to the fact that the phase of the photon OAM can vary by $m$ multiples of $2 \pi$ in an azimuthal rotation cycle $[10,11]$, whereby $m$ pairs of analyzers that have the property of $m$-fold rotational symmetry are used [ 8 9]. By contrast, the single-value period $\pi$ in the first case is attributed to the fact that the orientation of photon polarization only varies by $\pi$ in a rotation cycle, whereby two polarizers, a pair of analyzers of two-fold rotational symmetry, are used. The reasons for those single-value periods in the second and third cases are similar, whereby the respectively vital pair of analyzers are used. A variety of periods should be significant because a large period results in high angle resolution of the coincident fringe, whereas a small period results in a small range requirement of the relative orientation between the two analyzers.

Nevertheless, the OAM entanglement of twin photons obtained by SPDC can have infinite dimensions $[12,13]$. The higher dimension indicates that the violation is more robust against noise [14]. Dada et al. demonstrated violations of the generalized Bell inequality [15] with two-photon and up to 12-arbitrary-dimensional entanglement by using a setup with two spatial light modulations [16] that are configured by the hologram generation algorithm [17]. Oemrawsingh et al. demonstrated a violation of the Clauser-Horne-Shimony-Holt (CHSH) Bell inequality with two-photon and high-dimensional entanglement through two detections with two half-charge spiral phase plates (SPPs), and they obtained the outcomes of the coincidence fringe of the parabola and a strong Bell's parameter of $3 \frac{1}{5}[18,19]$. One key factor in verifying the quantum nonlocality with a parabolic coincidence fringe is that the quantum state of a light beam with fractional OAM (fractional optical vortex) is the superposition state consisting of numerous optical OAM eigenmodes [20] with a functional weight [21]. However, the periods of both of these coincident fringes are only $2 \pi$. In the latter case, the monotonic period is attributable to the rotational asymmetry of the SPP structure.

In this article, I theoretically demonstrate the overlap probability between two quantum states, each of which is a state with a rotational symmetry superposition made up of $n$ fractional OAM states, is $n$-section parabolas in a cycle round $2 \pi$. Various orthogonal relations exist between these two quantum states with charges of $\left(M-\frac{n}{2}\right) \bmod n=0$ and 
rotation angles of $\alpha=\pi(2 t-1) / n, t=1,2, \ldots, n$. The superposed quantum state has the property of $n$-fold rotational symmetry and is still the superposition state consisting of numerous optical OAM eigenmodes with functional weights [14]. I propose an experiment to prove the violations of the Bell inequality with high-dimensional two-particle OAM entanglement using an SPDC setup with two analyzers that have these two quantum states; however, the periods of the parabolic coincident fringes vary as $2 \pi / n$, and Bell's parameter of $3 \frac{1}{5}$ is obtained in each period. Thus, the requirement of small angle range of $2 \pi / n$ can be used to prove the quantum nonlocality with high-dimensional two-photon entanglement. These two analyzers consist of two $n$-section SPPs, and the period variable of parabolic coincident fringes is attributable to the fact that the structure of an $n$-section SPP has the property of $n$-fold rotational symmetry.

\section{THEORY}

An SPP with $n$ edge dislocations, denoted herein as SPP $n$, is an $n$-section SPP [22]. The $\mathrm{SPP} n$ structure has the property of $n$-fold rotational symmetry. Figure 1 shows the two cases of $n=2$ and 4, that is, SPP2 and SPP4, respectively. These are two- and four-section transparent dielectric plates, respectively, whose thicknesses vary linearly with the azimuthal angle $\phi$, and therefore, they shift the phases of the incident light fields in two- and four-fold symmetries, respectively. Without loss of generality, two indexes $M$ and $\alpha$ are used to respectively represent the thickness gradient with respect to $\phi$ and the orientation of the edge dislocation of an $\operatorname{SPP} n[18,21]$. Owing to the angle repetition for a rotation of $2 \pi, \alpha$ is bounded by $0 \leq \alpha<2 \pi$. An SPP $n$ can serve as an operator similar to the SPP in the basis set transformation for optical OAM eigenmodes in the Bell inequality experiment [18]. By operating an SPPn operator $\hat{S}_{n}(\alpha, M)$ on the integer OAM basis $\left\{\left|m^{\prime}\right\rangle\right\}$ [23], a new basis $\left\{\left|M n^{\left(m^{\prime}\right)}(\alpha)\right\rangle\right\}$ is expressed as $\hat{S}_{n}(\alpha, M)\left|m^{\prime}\right\rangle \equiv\left|M n^{\left(m^{\prime}\right)}(\alpha)\right\rangle$. The azimuthal part of a light field with OAM eigenvalue $m^{\prime}$ after this operation is $\left\langle\phi\left|\hat{S}_{n}(\alpha, M)\right| m^{\prime}\right\rangle=e^{i m^{\prime} \phi}\langle\phi \mid M n(\alpha)\rangle$. This is equal to one phase term $e^{i m^{\prime} \phi}$ times the other one $\langle\phi \mid M n(\alpha)\rangle$ that has the phase shift structure made from an SPP $n$. It is readily seen that $|M n(\alpha)\rangle$ is the quantum state of a light beam that is the exportation by passing a fundamental Gaussian light beam through an $\mathrm{SPP} n$. For $n=1$, by using an SPP, this is the quantum state of a fractional optical vortex with fractional topological charge $M,|M(\alpha)\rangle$ [21]. 

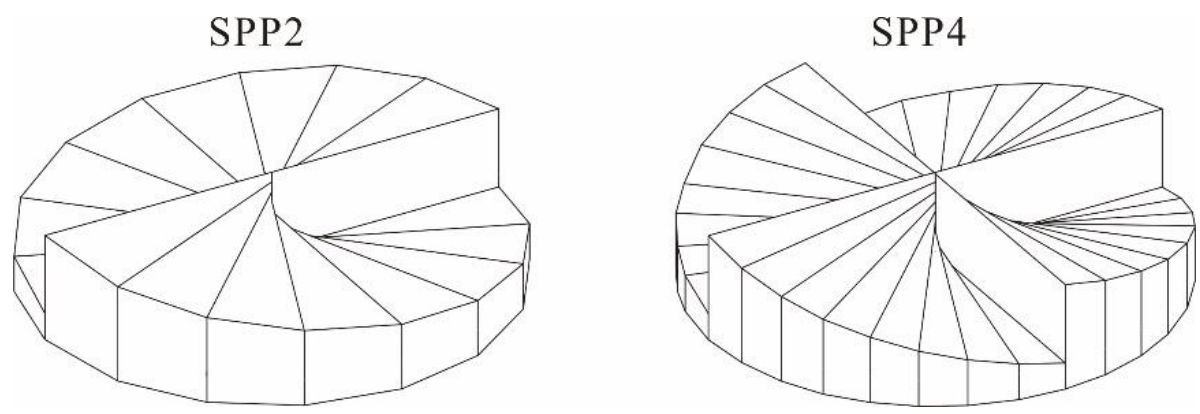

Fig. 1. Schemas of SPP2 and SPP4.

The phase gradients of the helical wavefront with respect to $\phi$ are invariant for azimuthally superposing fractional optical vortexes with identical charges [14] as well as that with integer charge $m$ [24]. The quantum state follows a similar principle; in having identical $M$, $|M n(\alpha)\rangle$ with $M$ must be given with the rotational symmetry superposition made up of $n$ fractional OAM states $|M(\alpha)\rangle$ with $M$ by

$$
|M n\rangle=\frac{\left|M n^{\prime}\right\rangle}{\sqrt{\left\langle M n^{\prime} \mid M n^{\prime}\right\rangle}},\left|M n^{\prime}\right\rangle=\sum_{k=0}^{n-1} \hat{U}\left(2 \pi \times \frac{k}{n}\right)|M\rangle,
$$

where $\hat{U}$ is the rotation operator, as introduced in Appendix $\mathrm{A}$, and $\left|M n^{\prime}\right\rangle$ is the unnormalized quantum state, whose field amplitudes with superposition of $n=2$ and 4 are derived in Appendix B. Along with the structure of $\operatorname{SPP} n,|M n(\alpha)\rangle$ has the property of $n$-fold rotational symmetry, which is proven theoretically in Appendix C, and the rotational symmetry of the structured light beams with $|M 2\rangle$ and $|M 4\rangle$ has been verified experimentally in terms of intensity image and phase profile in Ref. 14. $|M n(\alpha)\rangle$, similar to $|M(\alpha)\rangle[20,21]$, is a superposition state consisting of numerous optical OAM eigenmodes with functional weights [24], although $|M n(\alpha)\rangle$ has vanished OAM components as $m^{\prime} \bmod n$ [14]. A large $n$ implies a large rotational symmetry for both the structure of SPP $n$ and $|M n(\alpha)\rangle$. Let $\alpha=0$ and $\left\{\left|M n^{\left(m^{\prime}\right)}(0)\right\rangle\right\}$ be complete because $\hat{S}_{n}(\alpha, M)$ is a unitary operator (an invariant field state that is passing through two SPP $n$ s with identical $M$ and $n$ but handedness reversal). $\left|M n^{\left(m^{\prime}\right)}(\alpha)\right\rangle$ can be a superposition of these basis states, and $\alpha$ is 
equal to the intersection angle between two SPPns. Thus, the decomposition of $\left|M n^{\left(m^{\prime}\right)}(\alpha)\right\rangle$ into $\left\{\left|M n^{\left(m^{\prime}\right)}(0)\right\rangle\right\}$ depends on $\alpha$. The sum of these projection scalars is equal to the overlap amplitude $\langle\operatorname{Mn}(\alpha) \mid \operatorname{Mn}(0)\rangle$, whose modulus square, the overlap probability, is $|\langle M n(0) \mid M n(\alpha)\rangle|^{2}$. The formulas derived in Appendix D are used to calculate the overlap probability with $n$.

For $n=1$, the overlap probability between the quantum state of a light beam with fractional OAM and its rotation state with $\alpha$ is [18]

$$
|\langle M(0) \mid M(\alpha)\rangle|^{2}=\left(1-\frac{\alpha}{\pi}\right)^{2} \sin ^{2}(M \pi)+\cos ^{2}(M \pi), 0 \leq \alpha<2 \pi,
$$

where Eq. (A3) is used.

The overlap amplitude for $n=2$ is

$$
\langle M 2(0) \mid M 2(\alpha)\rangle=\frac{\langle M(0) \mid M(\alpha)\rangle+\langle M(0)|\hat{U}(\pi)| M(\alpha)\rangle}{1+\cos (\pi M)},
$$

where Eqs. (B1) and (D3) are used. The $n=2$ overlap probability is equal to the modulus square of the right hand side of Eq. (3) as

$$
\begin{aligned}
& |\langle M 2(0) \mid M 2(\alpha)\rangle|^{2}=\left\{|\langle M(0) \mid M(\alpha)\rangle|^{2}+|\langle M(0)|\hat{U}(\pi)| M(\alpha)\rangle|^{2}\right. \\
& \left.+2 \operatorname{Re}\left[\langle M(0) \mid M(\alpha)\rangle\langle M(0)|\hat{U}(\pi)| M(\alpha)\rangle^{*}\right]\right\} /[1+\cos (\pi M)]^{2}
\end{aligned}
$$

Let $\beta=\pi$ in Eqs. (D7) and (D5), which are respectively expressed as

$$
|\langle M(0)|\hat{U}(\pi)| M(\alpha)\rangle|^{2}=\frac{1}{\pi^{2}} \times\left\{\begin{array}{l}
\left(\alpha^{2}-\pi^{2}\right) \sin ^{2}(M \pi)+\pi^{2}, 0 \leq \alpha<\pi \\
(\alpha-\pi)(\alpha-3 \pi) \sin ^{2}(M \pi)+\pi^{2}, \pi \leq \alpha<2 \pi
\end{array}\right.
$$

and

$$
\begin{aligned}
& \operatorname{Re}\left[\langle M(0) \mid M(\alpha)\rangle\langle M(0)|\hat{U}(\pi)| M(\alpha)\rangle^{*}\right] \\
& =\frac{1}{4 \pi^{2}} \times\left\{\begin{array}{l}
\alpha(\pi-\alpha) \cos (3 M \pi)+\left(4 \pi^{2}-\pi \alpha+\alpha^{2}\right) \cos (M \pi), 0 \leq \alpha<\pi \\
(2 \pi-\alpha)(\alpha-\pi) \cos (3 M \pi)+\left(6 \pi^{2}-3 \pi \alpha+\alpha^{2}\right) \cos (M \pi), \pi \leq \alpha<2 \pi
\end{array}\right.
\end{aligned}
$$

Substituting Eqs. (2), (5), and (6) into Eq. (4) gives 


$$
|\langle M 2(0) \mid M 2(\alpha)\rangle|^{2}=\left\{\begin{array}{l}
\left(1-\frac{2 \alpha}{\pi}\right)^{2} \sin ^{2}\left(\frac{M \pi}{2}\right)+\cos ^{2}\left(\frac{M \pi}{2}\right), 0 \leq \alpha<\pi \\
\left(3-\frac{2 \alpha}{\pi}\right)^{2} \sin ^{2}\left(\frac{M \pi}{2}\right)+\cos ^{2}\left(\frac{M \pi}{2}\right), \pi \leq \alpha<2 \pi
\end{array} .\right.
$$

The overlap amplitude for $n=4$ is

$$
\begin{aligned}
\langle M 4(0) \mid M 4(\alpha)\rangle= & {\left[\langle M(0) \mid M(\alpha)\rangle+\left\langle M(0)\left|\hat{U}\left(\frac{\pi}{2}\right)\right| M(\alpha)\right\rangle+\langle M(0)|\hat{U}(\pi)| M(\alpha)\rangle\right.} \\
& \left.+\left\langle M(0)\left|\hat{U}\left(\frac{3 \pi}{2}\right)\right| M(\alpha)\right\rangle\right] /\left[1+\cos (\pi M)+2 \cos ^{3}\left(\frac{\pi}{2} M\right)\right],
\end{aligned}
$$

where Eqs. (B2) and (D3) are used. The $n=4$ overlap probability is equal to the modulus square of the right hand side of Eq. (8) as

$$
\begin{aligned}
& |\langle M 4(0) \mid M 4(\alpha)\rangle|^{2}=\left\{|\langle M(0) \mid M(\alpha)\rangle|^{2}+|\langle M(0)|\hat{U}(\pi)| M(\alpha)\rangle|^{2}+\left|\left\langle M(0)\left|\hat{U}\left(\frac{\pi}{2}\right)\right| M(\alpha)\right\rangle\right|^{2}\right. \\
& +\left|\left\langle M(0)\left|\hat{U}\left(\frac{3 \pi}{2}\right)\right| M(\alpha)\right\rangle\right|^{2}+2 \operatorname{Re}\left[\langle M(0) \mid M(\alpha)\rangle\langle M(0)|\hat{U}(\pi)| M(\alpha)\rangle^{*}\right] \\
& +2 \operatorname{Re}\left[\langle M(0) \mid M(\alpha)\rangle\left\langle M(0)\left|\hat{U}\left(\frac{\pi}{2}\right)\right| M(\alpha)\right\rangle^{*}\right]+2 \operatorname{Re}\left[\langle M(0) \mid M(\alpha)\rangle\left\langle M(0)\left|\hat{U}\left(\frac{3 \pi}{2}\right)\right| M(\alpha)\right\rangle^{*}\right] \\
& +2 \operatorname{Re}\left[\langle M(0)|\hat{U}(\pi)| M(\alpha)\rangle\left\langle M(0)\left|\hat{U}\left(\frac{\pi}{2}\right)\right| M(\alpha)\right\rangle^{*}\right]+2 \operatorname{Re}\left[\langle M(0)|\hat{U}(\pi)| M(\alpha)\rangle\left\langle M(0)\left|\hat{U}\left(\frac{3 \pi}{2}\right)\right| M(\alpha)\right\rangle^{*}\right] \\
& \left.+2 \operatorname{Re}\left[\left\langle M(0)\left|\hat{U}\left(\frac{\pi}{2}\right)\right| M(\alpha)\right\rangle\left\langle M(0)\left|\hat{U}\left(\frac{3 \pi}{2}\right)\right| M(\alpha)\right\rangle^{*}\right]\right\} /\left[1+\cos (\pi M)+2 \cos ^{3}\left(\frac{\pi}{2} M\right)\right]^{2} .
\end{aligned}
$$

Substituting $\beta=\pi / 2$ and $3 \pi / 2$ in Eq. (D7) gives

$$
\left|\left\langle M(0)\left|\hat{U}\left(\frac{\pi}{2}\right)\right| M(\alpha)\right\rangle\right|^{2}=\frac{1}{\pi^{2}} \times\left\{\begin{array}{l}
\left(\alpha+\frac{\pi}{2}\right)\left(\alpha-\frac{3 \pi}{2}\right) \sin ^{2}(M \pi)+\pi^{2}, 0 \leq \alpha<\frac{3 \pi}{2} \\
\left(\alpha-\frac{3 \pi}{2}\right)\left(\alpha-\frac{7 \pi}{2}\right) \sin ^{2}(M \pi)+\pi^{2}, \frac{3 \pi}{2} \leq \alpha<2 \pi
\end{array}\right.
$$

and

$$
\left|\left\langle M(0)\left|\hat{U}\left(\frac{3 \pi}{2}\right)\right| M(\alpha)\right\rangle\right|^{2}=\frac{1}{\pi^{2}} \times\left\{\begin{array}{l}
\left(\alpha+\frac{3 \pi}{2}\right)\left(\alpha-\frac{\pi}{2}\right) \sin ^{2}(M \pi)+\pi^{2}, 0 \leq \alpha<\frac{\pi}{2} \\
\left(\alpha-\frac{\pi}{2}\right)\left(\alpha-\frac{5 \pi}{2}\right) \sin ^{2}(M \pi)+\pi^{2}, \frac{\pi}{2} \leq \alpha<2 \pi
\end{array} .\right.
$$

Substituting $\beta=\pi / 2$ and $3 \pi / 2$ in Eq. (D5) gives

$$
\begin{aligned}
& \operatorname{Re}\left[\langle M(0) \mid M(\alpha)\rangle\left\langle M(0)\left|\hat{U}\left(\frac{\pi}{2}\right)\right| M(\alpha)\right\rangle^{*}\right] \\
& =\frac{1}{4 \pi^{2}} \times\left\{\begin{array}{l}
{\left[2\left(2 \alpha^{2}-3 \pi \alpha-\pi^{2}\right) \sin ^{2}(M \pi)+4 \pi^{2}\right] \cos \left(\frac{M \pi}{2}\right)+\pi^{2} \sin (2 M \pi) \sin \left(\frac{M \pi}{2}\right), 0 \leq \alpha<\frac{3 \pi}{2}} \\
\left(2 \alpha^{2}-7 \pi \alpha+7 \pi^{2}\right) \cos \left(\frac{3 M \pi}{2}\right)+\left(\frac{7}{2} \pi \alpha-\alpha^{2}-3 \pi^{2}\right) \cos \left(\frac{7 M \pi}{2}\right)+\alpha\left(\frac{7}{2} \pi-\alpha\right) \cos \left(\frac{M \pi}{2}\right), \frac{3 \pi}{2} \leq \alpha<2 \pi
\end{array}\right.
\end{aligned}
$$

and 


$$
\begin{aligned}
& \operatorname{Re}\left[\langle M(0) \mid M(\alpha)\rangle\left\langle M(0)\left|\hat{U}\left(\frac{3 \pi}{2}\right)\right| M(\alpha)\right\rangle^{*}\right] \\
& =\frac{1}{4 \pi^{2}} \times\left\{\begin{array}{l}
{\left[2\left(2 \alpha^{2}-\pi \alpha+3 \pi^{2}\right) \sin ^{2}(M \pi)+4 \pi^{2}\right] \cos \left(\frac{3 M \pi}{2}\right)+3 \pi^{2} \sin (2 M \pi) \sin \left(\frac{3 M \pi}{2}\right), 0 \leq \alpha<\frac{\pi}{2}} \\
\left(2 \alpha^{2}-5 \pi \alpha+5 \pi^{2}\right) \cos \left(\frac{M \pi}{2}\right)+\left(\frac{5}{2} \pi \alpha-\alpha^{2}-\pi^{2}\right) \cos \left(\frac{5 M \pi}{2}\right)+\alpha\left(\frac{5}{2} \pi-\alpha\right) \cos \left(\frac{3 M \pi}{2}\right), \frac{\pi}{2} \leq \alpha<2 \pi
\end{array} .\right.
\end{aligned}
$$

Substituting $\beta_{1}=\pi$ and $\beta_{2}=\pi / 2, \beta_{1}=\pi$ and $\beta_{2}=3 \pi / 2$, and $\beta_{1}=\pi / 2$ and $\beta_{2}=3 \pi / 2$ in Eq. (D8) gives

$$
\begin{aligned}
& \operatorname{Re}\left[\langle M(0)|\hat{U}(\pi)| M(\alpha)\rangle\left\langle M(0)\left|\hat{U}\left(\frac{\pi}{2}\right)\right| M(\alpha)\right\rangle^{*}\right]= \\
& \frac{1}{4 \pi^{2}} \times\left\{\begin{array}{l}
{\left[4 \sin ^{2}(M \pi)\left(\alpha^{2}-\frac{\pi}{2} \alpha-\pi^{2}\right)+4 \pi^{2}\right] \cos \left(\frac{M \pi}{2}\right)+\pi^{2} \sin (2 M \pi) \sin \left(\frac{M \pi}{2}\right), 0 \leq \alpha<\pi} \\
\left(2 \alpha^{2}+4 \pi^{2}-5 \pi \alpha\right) \cos \left(\frac{3 M \pi}{2}\right)+(\alpha-\pi)\left(\frac{3 \pi}{2}-\alpha\right) \cos \left(\frac{7 M \pi}{2}\right)+(3 \pi-\alpha)\left(\alpha+\frac{\pi}{2}\right) \cos \left(\frac{M \pi}{2}\right), \pi \leq \alpha<\frac{3 \pi}{2}, \\
{\left[4 \sin ^{2}(M \pi)\left(\alpha^{2}-\frac{9}{2} \pi \alpha+4 \pi^{2}\right)+4 \pi^{2}\right] \cos \left(\frac{M \pi}{2}\right)+\pi^{2} \sin (2 M \pi) \sin \left(\frac{M \pi}{2}\right), \frac{3 \pi}{2} \leq \alpha<2 \pi}
\end{array}\right.
\end{aligned}
$$

$$
\begin{aligned}
& \operatorname{Re}\left[\langle M(0)|\hat{U}(\pi)| M(\alpha)\rangle\left\langle M(0)\left|\hat{U}\left(\frac{3}{2} \pi\right)\right| M(\alpha)\right\rangle^{*}\right]= \\
& \frac{1}{4 \pi^{2}} \times\left\{\begin{array}{l}
{\left[4 \sin ^{2}(M \pi)\left(\alpha^{2}+\frac{\pi}{2} \alpha-\pi^{2}\right)+4 \pi^{2}\right] \cos \left(\frac{M \pi}{2}\right)+\pi^{2} \sin (2 M \pi) \sin \left(\frac{M \pi}{2}\right), 0 \leq \alpha<\frac{\pi}{2}} \\
\left(2 \alpha^{2}+2 \pi^{2}-3 \pi \alpha\right) \cos \left(\frac{3 M \pi}{2}\right)+(\pi-\alpha)\left(\alpha-\frac{\pi}{2}\right) \cos \left(\frac{7 M \pi}{2}\right)+(\alpha+\pi)\left(\frac{5 \pi}{2}-\alpha\right) \cos \left(\frac{M \pi}{2}\right), \frac{\pi}{2} \leq \alpha<\pi \\
{\left[4 \sin ^{2}(M \pi)\left(\alpha^{2}-\frac{7 \pi}{2} \alpha+2 \pi^{2}\right)+4 \pi^{2}\right] \cos \left(\frac{M \pi}{2}\right)+\pi^{2} \sin (2 M \pi) \sin \left(\frac{M \pi}{2}\right), \pi \leq \alpha<2 \pi}
\end{array}\right.
\end{aligned}
$$

, and

$$
\begin{aligned}
& \operatorname{Re}\left[\left\langle M(0)\left|\hat{U}\left(\frac{\pi}{2}\right)\right| M(\alpha)\right\rangle\left\langle M(0)\left|\hat{U}\left(\frac{3 \pi}{2}\right)\right| M(\alpha)\right\rangle^{*}\right]= \\
& \frac{1}{4 \pi^{2}} \times\left\{\begin{array}{l}
{\left[4 \sin ^{2}(M \pi)\left(\alpha^{2}-\frac{5}{4} \pi^{2}\right)+4 \pi^{2}\right] \cos (M \pi)+2 \pi^{2} \sin (2 M \pi) \sin (M \pi), 0 \leq \alpha<\frac{\pi}{2}} \\
\left(\alpha^{2}-2 \pi \alpha+\frac{19}{4} \pi^{2}\right) \cos (M \pi)+\left(\frac{3 \pi}{2}-\alpha\right)\left(\alpha-\frac{\pi}{2}\right) \cos (3 M \pi), \frac{\pi}{2} \leq \alpha<\frac{3 \pi}{2} \\
{\left[4 \sin ^{2}(M \pi)\left(\alpha^{2}-4 \pi \alpha+\frac{11}{4} \pi^{2}\right)+4 \pi^{2}\right] \cos (M \pi)+2 \pi^{2} \sin (2 M \pi) \sin (M \pi), \frac{3 \pi}{2} \leq \alpha<2 \pi}
\end{array} .\right.
\end{aligned}
$$

Substituting Eqs. (2), (5), (6), and (10)-(16) into Eq. (9) gives 


$$
|\langle M 4(0) \mid M 4(\alpha)\rangle|^{2}=\left\{\begin{array}{l}
\left(1-\frac{4 \alpha}{\pi}\right)^{2} \sin ^{2}\left(\frac{M \pi}{4}\right)+\cos ^{2}\left(\frac{M \pi}{4}\right), 0 \leq \alpha<\frac{\pi}{2} \\
\left(3-\frac{4 \alpha}{\pi}\right)^{2} \sin ^{2}\left(\frac{M \pi}{4}\right)+\cos ^{2}\left(\frac{M \pi}{4}\right), \frac{\pi}{2} \leq \alpha<\pi \\
\left(5-\frac{4 \alpha}{\pi}\right)^{2} \sin ^{2}\left(\frac{M \pi}{4}\right)+\cos ^{2}\left(\frac{M \pi}{4}\right), \pi \leq \alpha<\frac{3 \pi}{2} \\
\left(7-\frac{4 \alpha}{\pi}\right)^{2} \sin ^{2}\left(\frac{M \pi}{4}\right)+\cos ^{2}\left(\frac{M \pi}{4}\right), \frac{3 \pi}{2} \leq \alpha<2 \pi
\end{array} .\right.
$$

According to Eqs. (2), (7), and (17), the overlap probability for arbitrary $n$ should be calculated as

$$
\begin{gathered}
|\langle M n(0) \mid M n(\alpha)\rangle|^{2}=\frac{[\pi(2 t-1)-n \alpha]^{2}}{\pi^{2}} \sin ^{2}\left(\frac{M \pi}{n}\right)+\cos ^{2}\left(\frac{M \pi}{n}\right), \\
\frac{2 \pi}{n}(t-1) \leq \alpha<\frac{2 \pi}{n} t, t=1,2, \ldots, n .
\end{gathered}
$$

This probability generally depends on the integer part of the step index $m$; however, it is independent of the topological charge of twin photons $\mathrm{m}^{\prime}$. In particular, it is independent of $m$ and $m^{\prime}$ as $n=1$ [18]. Eq. (18) is a constant function of $\alpha$ as $M \bmod n=0$, and it is otherwise a parabolic function of $\alpha$ that is repeated between $n$ sections as $M \bmod n \neq 0$. These $n$-section parabolic functions, similar to previously reported parabolic functions [18], indicates that SPPn rotates the OAM state following through the Hilbert space that is not confined to a two-dimensional subspace. There are orthogonal relations such as $\left(M-\frac{n}{2}\right) \bmod n=0$ at $\alpha=\pi(2 t-1) / n$. Equivalently, all the total phase variations in each fold of these superposed quantum states with various $n$ cases are module $2 \pi$ by $\pi$ in these orthogonal relations. Two probability functions with $n=2$ and $n=4$ are plotted in Fig. 2 for cases of uncorrelated, correlated, and orthogonal relations.

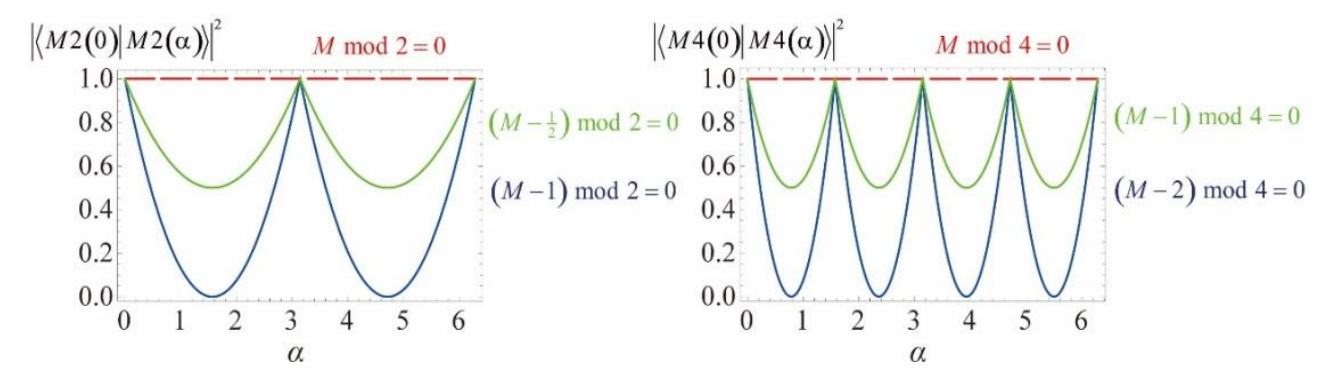

Fig. 2. Various angle periods of overlap probability. Left: three overlap probabilities between a superposed quantum state $|M 2(0)\rangle$ and its rotation $|M 2(\alpha)\rangle$ in the charges $M \bmod 2=0$, $\left(M-\frac{1}{2}\right) \bmod 2=0$, and $(M-1) \bmod 2=0$, as indicated respectively by red dotted, green, and blue solid curves. The first is a constant, whereas the latter two are parabolic functions of 
$\alpha$ down to 0.5 and 0 at $\alpha=\pi / 2$ and $3 \pi / 2$. Right: three overlap probabilities between a superposed quantum state $|M 4(0)\rangle$ and its rotation $|M 4(\alpha)\rangle$ in the charges $M \bmod 4=0$, $(M-1) \bmod 4=0$, and $(M-2) \bmod 4=0$. The first is a constant, whereas the latter two are parabolic functions of $\alpha$ down to 0.5 and 0 at $\alpha=\pi / 4,3 \pi / 4,5 \pi / 4$, and $7 \pi / 4$.

\section{PROPOSED EXPERIMENT}

I propose an experimental setup similar to the one in Figure 2 of Ref. 18 but with the two SPPs with half-integers being replaced by two SPP $n$ s with $\left(M-\frac{n}{2}\right) \bmod n=0$ to prove the violations of the Bell inequality with high-dimensional two-particle OAM entanglement. This is a type of SPDC setup. Its generated twin photons in two arms, signal and idler, obey OAM conservation [12] provided that two conditions of the paraxial limit and thin-crystal approximation [25-28]. By choosing a fundamental Gaussian pump beam in an SPDC setup with photon OAM conservation, the normalized coincidence fringe detected by the two photons after passing two $\operatorname{SPP} n$ s that are intersected by $\alpha$ is equal to the mode overlap probability between two quantum states (Eq. (18)), as also described in Refs. 18 and 19. However, the fringes are $n$-section parabolas. These expected outcomes with various azimuthal angle periods of parabolic coincident fringes will have a small range of intersection angles in proving quantum nonlocality with high-dimensional two-photon entanglement.

As in the case of two SPPs [18], the CHSH inequality is chosen in my proposed experiment as $S=E\left(\alpha_{s}, \alpha_{i}\right)-E\left(\alpha_{s}^{\prime}, \alpha_{i}\right)+E\left(\alpha_{s}, \alpha_{i}^{\prime}\right)+E\left(\alpha_{s}^{\prime}, \alpha_{i}^{\prime}\right) \leq 2 \quad$ [4], where $s$ and $i$ denote the signal and idler in the SPDC experiment, respectively. $E$ is expressed as $[5,29]$

$$
E\left(\alpha_{s}, \alpha_{i}\right)=\frac{P\left(\alpha_{s}, \alpha_{i}\right)+P\left(\alpha_{s}^{\perp}, \alpha_{i}^{\perp}\right)-P\left(\alpha_{s}, \alpha_{i}^{\perp}\right)-P\left(\alpha_{s}^{\perp}, \alpha_{i}\right)}{P\left(\alpha_{s}, \alpha_{i}\right)+P\left(\alpha_{s}^{\perp}, \alpha_{i}^{\perp}\right)+P\left(\alpha_{s}, \alpha_{i}^{\perp}\right)+P\left(\alpha_{s}^{\perp}, \alpha_{i}\right)}
$$

where $P\left(\alpha_{s}, \alpha_{i}\right)$ is the coincidence probability function proportional to $\left[1-\left|\alpha_{s}-\alpha_{i}\right| /(\pi / n)\right]^{2}$ in the orthogonal case of $\left(M-\frac{n}{2}\right) \bmod n=0$ and only the modulus of $\alpha_{s}-\alpha_{i} . \quad \alpha_{s}^{\perp}$ and $\alpha_{i}^{\perp}$ are the angles of two analyzers for the states orthogonal to the states with setting $\alpha_{s}$ and $\alpha_{i}$, respectively. In this case, there are $n$ pairs of them as $\alpha_{s}^{\perp} \equiv \alpha_{s}+\pi(2 t-1) / n$ and $\alpha_{i}^{\perp} \equiv \alpha_{i}+\pi(2 t-1) / n, t=1,2, \ldots, n$. As the periodicity in this case is $1 / 2 n$ that in the case of photon polarization entanglement, I use the standard analyzer settings of a previous case [5, 30] divided by $2 n: \alpha_{s}=-\pi / 4 n, \alpha_{s}^{\prime}=\pi / 4 n, \alpha_{i}=-\pi / 2 n$, and $\alpha_{i}=0$. Upon substituting these parameters into Eq. (19), Bell's parameter in each of the $n$-sections can be evaluated as $3 \frac{1}{5}$, which is identical to that mentioned in Ref. 18 .

\section{CONCLUSION}


I have theoretically demonstrated the overlap probability between two quantum states, each of which is a state with a rotational symmetry superposition made up of $n$ fractional OAM states, is $n$-section parabolas. There are various orthogonal relations between these two quantum states at charges of $\left(M-\frac{n}{2}\right) \bmod n=0$ and relative rotation angle $\alpha=\pi(2 t-1) / n, t=1,2, \ldots, n$. I propose to prove the violations of the Bell inequality with high-dimensional two-particle OAM entanglement in an SPDC experiment with two SPP $n$ s where the intersection angle represents various periods of parabolic coincident fringes. The occurring charge of $\left(M-\frac{n}{2}\right) \bmod n=0$ is coincident with the occurrence of the completely destructive interference [24] (cf. Ref. 24, except $M=1 / 2$ ) and maximum phase dislocation degree for a light beam with phase singularity that is characterized with azimuthally symmetric angular positions [14]. 


\section{APPENDIX A: QUANTUM STATE OF A LIGHT BEAM WITH FRACTIONAL OAM}

The quantum state of a light beam with fractional OAM is denoted by $|M(\alpha)\rangle$ [21], where $M=m+\mu$ and the parameter $\alpha$, bounded by $0 \leq \alpha<2 \pi$, is the angular position of the discontinuity. A function $f_{\alpha}(\phi)$ is introduced as

$$
f_{\alpha}(\phi)=\left\{\begin{array}{l}
1,0 \leq \phi<\alpha \\
0, \alpha \leq \phi<2 \pi
\end{array} .\right.
$$

By using Eq. (A1), a definition can be shown as

$$
\langle\phi \mid M(\alpha)\rangle \equiv e^{i m \phi} e^{i \mu\left[\phi+2 \pi f_{\alpha}(\phi)-\alpha\right]} .
$$

Based on the completeness relation and Eq. (A2), the overlap amplitude between two states with an intersection angle $\alpha$ is

$$
\begin{aligned}
\langle M(0) \mid M(\alpha)\rangle & =\frac{1}{2 \pi} \int_{0}^{2 \pi} d \phi e^{i 2 \pi \mu\left[f_{\alpha}(\phi)-f_{0}(\phi)\right]} e^{i \mu(0-\alpha)} \\
& =\frac{1}{2 \pi}\left[\alpha e^{i(2 \pi-\alpha) \mu}+(2 \pi-\alpha) e^{-i \alpha \mu}\right] .
\end{aligned}
$$

$|M(\alpha)\rangle$ can be decomposed into integer OAM eigenmodes, the probability distribution of which can be obtained by setting $\langle M(0)|=\left\langle m^{\prime}\right|$ in Eq. (A3)

$$
c_{m^{\prime}}[M(\alpha)]=\left\langle m^{\prime} \mid M(\alpha)\right\rangle=\frac{i e^{i\left(m-m^{\prime}\right) \alpha}}{2 \pi\left(M-m^{\prime}\right)}\left(1-e^{i 2 \pi \mu}\right)
$$

The state resulting from a unitary operator $\hat{U}(\beta)$ is the effect of the rotation for this quantum state and an additional phase term $e^{-i m \beta}$ :

$$
\hat{U}(\beta)|M(\alpha)\rangle=e^{-i m \beta}|M(\alpha \oplus \beta)\rangle,
$$

where the parameter $\beta$ bounded by $0 \leq \beta<2 \pi$ is the action on the state and $\alpha \oplus \beta=(\alpha+\beta) \bmod 2 \pi$ yields a result in the range $[0,2 \pi)$ owing to the $2 \pi$ modulo. The multiplication of rotation operators has the combination characteristic

$$
\hat{U}\left(\beta_{1}\right) \hat{U}\left(\beta_{2}\right)|M(\alpha)\rangle=\hat{U}\left(\beta_{1} \oplus \beta_{2}\right)|M(\alpha)\rangle .
$$

Some useful formulas are derived as follows. 


$$
\langle M(\alpha)|\hat{U}(\beta)| M(\alpha)\rangle=\frac{e^{-i m \beta}}{2 \pi}\left[\beta e^{i(2 \pi-\beta) \mu}+(2 \pi-\beta) e^{-i \beta \mu}\right],
$$

where Eqs. (A3) and (A5) are used. The real part of Eq. (A7) is

$$
\operatorname{Re}\langle M(\alpha)|\hat{U}(\beta)| M(\alpha)\rangle=\frac{1}{2 \pi}[\beta \cos (2 \pi \mu-\beta M)+(2 \pi-\beta) \cos (\beta M)] .
$$

By using Eqs. (A4) and (A5),

$$
\left\langle m^{\prime}|\hat{U}(\beta)| M(\alpha)\right\rangle=e^{-i m \beta} \frac{i e^{i\left(m-m^{\prime}\right)(\beta \oplus \alpha)}}{2 \pi\left(M-m^{\prime}\right)}\left(1-e^{i 2 \pi \mu}\right) .
$$

\section{APPENDIX B: USEFUL FORMULAS FOR QUANTUM STATES WITH SUPERPOSITIONS OF $n=2$ AND 4}

In Eq. (1), the field amplitude of the unnormalized quantum state with $n=2$ superposition is

$$
\begin{aligned}
\left\langle M 2^{\prime} \mid M 2^{\prime}\right\rangle & =\left[\left\langleM\left|+\langle M| \hat{U}^{\dagger}(\pi)\right][|M\rangle+\hat{U}(\pi)|M\rangle]\right.\right. \\
& =2[\langle M \mid M\rangle+\operatorname{Re}\langle M|\hat{U}(\pi)| M\rangle]=2[1+\cos (M \pi)],
\end{aligned}
$$

where the unitary property of $\hat{U}, \cos (2 \pi \mu-\pi M)=\cos (\pi M-2 \pi m)=\cos (\pi M)$, and Eqs. (A3) and (A8) are used. The field amplitude of the unnormalized quantum state with $n=4$ superposition in Eq. (1) is

$$
\begin{aligned}
\left\langle M 4^{\prime} \mid M 4^{\prime}\right\rangle= & \left\{\left[\langle M|+\langle M| \hat{U}^{\dagger}\left(\frac{\pi}{2}\right)+\langle M| \hat{U}^{\dagger}(\pi)+\langle M| \hat{U}^{\dagger}\left(\frac{3 \pi}{2}\right)\right]\right. \\
& {\left.\left[|M\rangle+\hat{U}\left(\frac{\pi}{2}\right)|M\rangle+\hat{U}(\pi)|M\rangle+\hat{U}\left(\frac{3 \pi}{2}\right)|M\rangle\right]\right\} } \\
= & 4\left[\langle M \mid M\rangle+\operatorname{Re}\left\langle M\left|\hat{U}\left(\frac{\pi}{2}\right)\right| M\right\rangle+\operatorname{Re}\langle M|\hat{U}(\pi)| M\rangle+\operatorname{Re}\left\langle M\left|\hat{U}\left(\frac{3 \pi}{2}\right)\right| M\right\rangle\right] \\
= & 4+4 \cos (\pi M)+8 \cos ^{3}\left(\frac{\pi}{2} M\right),
\end{aligned}
$$

where the unitary property of $\hat{U}, e^{-i 2 \pi m}=1$, and Eqs. (A3), (A6), and (A8) are used.

\section{APPENDIX C: ROTATIONAL SYMMETRY OF $|M n(\alpha)\rangle$}

I describe the order of rotational symmetry for $|M n(\alpha)\rangle$. For $n=1,|M\rangle$ has the property of least rotational symmetry of one-fold $C_{1}$ considering its rotation by $\theta=2 \pi$ as

$$
\hat{U}(2 \pi)|M\rangle=|M\rangle .
$$

$|M\rangle$ does not have rotational symmetry with order larger than one because of the innate 
character of the phase structure of a fractional optical vortex. The rotational symmetry of $|M n(\alpha)\rangle, n>1$, can be verified by the state with the superposition made up of the original and rotated states. For $n=2,\left|M 2^{\prime}\right\rangle \equiv \hat{U}(\pi)|M\rangle+|M\rangle$ from Eq. (1). $\left|M 2^{\prime}\right\rangle$ satisfies the least rotational symmetry as

$$
\hat{U}(2 \pi)\left|M 2^{\prime}\right\rangle=\left|M 2^{\prime}\right\rangle
$$

To sum $\left|M 2^{\prime}\right\rangle$ and its rotation state with $\theta=\pi$, I have

$$
\begin{aligned}
& \hat{U}(\pi)\left|M 2^{\prime}\right\rangle+\left|M 2^{\prime}\right\rangle=\hat{U}(\pi) \hat{U}(\pi)|M\rangle+\hat{U}(\pi)|M\rangle+\hat{U}(\pi)|M\rangle+|M\rangle= \\
& 2[\hat{U}(\pi)|M\rangle+|M\rangle]=2\left|M 2^{\prime}\right\rangle,
\end{aligned}
$$

where Eq. (A8) and Eq. (C1) are used. From Eq. (C3),

$$
\hat{U}(\pi)\left|M 2^{\prime}\right\rangle=\left|M 2^{\prime}\right\rangle
$$

Owing to Eqs. (C2) and (C4), $\left|M 2^{\prime}\right\rangle$ has the property of rotational symmetry of $C_{2}$. $\left|M 2^{\prime}\right\rangle \equiv \hat{U}(\pi)|M\rangle+|M\rangle$ does not have the rotational symmetry with order larger than two, because $|M\rangle$ does not have rotational symmetry with order larger than one.

For $n=4,\left|M 4^{\prime}\right\rangle \equiv|M\rangle+\hat{U}\left(\frac{\pi}{2}\right)|M\rangle+\hat{U}(\pi)|M\rangle+\hat{U}\left(\frac{3 \pi}{2}\right)|M\rangle$, from Eq. (1). $\left|M 4^{\prime}\right\rangle$ satisfies the least rotational symmetry as

$$
\hat{U}(2 \pi)\left|M 4^{\prime}\right\rangle=\left|M 4^{\prime}\right\rangle
$$

To sum $\left|M 4^{\prime}\right\rangle$ and its rotation with $\theta=\pi / 2$, I have

$$
\begin{aligned}
& \left|M 4^{\prime}\right\rangle+\hat{U}\left(\frac{\pi}{2}\right)\left|M 4^{\prime}\right\rangle=|M\rangle+\hat{U}\left(\frac{\pi}{2}\right)|M\rangle+\hat{U}(\pi)|M\rangle+\hat{U}\left(\frac{3 \pi}{2}\right)|M\rangle+ \\
& \hat{U}\left(\frac{\pi}{2}\right)|M\rangle+\hat{U}\left(\frac{\pi}{2}\right) \hat{U}\left(\frac{\pi}{2}\right)|M\rangle+\hat{U}\left(\frac{\pi}{2}\right) \hat{U}(\pi)|M\rangle+\hat{U}\left(\frac{\pi}{2}\right) \hat{U}\left(\frac{3 \pi}{2}\right)|M\rangle= \\
& 2\left[|M\rangle+\hat{U}\left(\frac{\pi}{2}\right)|M\rangle+\hat{U}(\pi)|M\rangle+\hat{U}\left(\frac{3 \pi}{2}\right)|M\rangle\right]=2\left|M 4^{\prime}\right\rangle
\end{aligned}
$$

where Eq. (A8) and Eq. (C1) are used. From Eq. (C6),

$$
\hat{U}\left(\frac{\pi}{2}\right)\left|M 4^{\prime}\right\rangle=\left|M 4^{\prime}\right\rangle
$$

Similarly, to sum $\left|M 4^{\prime}\right\rangle$ and its rotations with $\theta=\pi$ and $\theta=3 \pi / 2$, I have respectively

$$
\hat{U}(\pi)\left|M 4^{\prime}\right\rangle=\left|M 4^{\prime}\right\rangle,
$$

and

$$
\hat{U}\left(\frac{3 \pi}{2}\right)\left|M 4^{\prime}\right\rangle=\left|M 4^{\prime}\right\rangle
$$


Owing to Eqs. (C5), (C7)-(C9), $\left|M 4^{\prime}\right\rangle$ has the property of rotational symmetry of $C_{4}$. Similarly, $|M n\rangle$ has the property of rotational symmetry of $C_{n}$ by $\hat{U}(\theta=2 \pi \times t / n)|M n\rangle=|M n\rangle, t=1,2, \ldots, n$. The experimental superposition can be performed using a Mach-Zehnder interferometer (MZI) with $\theta$, and it has already been verified experimentally for two light beams whose quantum states are $|M 2\rangle$ and $|M 4\rangle$ [14].

\section{APPENDIX D: USEFUL FORMULAS FOR $|\langle\operatorname{Mn}(0) \mid \operatorname{Mn}(\alpha)\rangle|^{2}$}

$$
\begin{gathered}
\alpha \text { and } \beta \in[0,2 \pi) . \\
\langle M(0)|\hat{U}(\beta)| M(\alpha)\rangle=\frac{e^{-i m \beta}}{2 \pi} \times\left\{\begin{array}{l}
(\alpha+\beta) e^{i(2 \pi-\alpha-\beta) \mu}+(2 \pi-\alpha-\beta) e^{-i(\alpha+\beta) \mu}, \alpha+\beta \leq 2 \pi \\
(\alpha+\beta-2 \pi) e^{i(4 \pi-\alpha-\beta) \mu}+(4 \pi-\alpha-\beta) e^{-i(\alpha+\beta-2 \pi) \mu}, \alpha+\beta>2 \pi
\end{array}\right.
\end{gathered}
$$

, where Eqs. (A3) and (A5) are used. There are two expressions in two sections of Eq. (D1). Similarly,

$$
\langle M(0)|\hat{U}(2 \pi-\beta)| M(\alpha)\rangle=\frac{e^{i m \beta}}{2 \pi} \times\left\{\begin{array}{l}
(2 \pi+\alpha-\beta) e^{i(\beta-\alpha) \mu}+(\beta-\alpha) e^{-i(2 \pi+\alpha-\beta) \mu}, \alpha \leq \beta \\
(\alpha-\beta) e^{i(2 \pi+\beta-\alpha) \mu}+(2 \pi+\beta-\alpha) e^{-i(\alpha-\beta) \mu}, \alpha>\beta
\end{array}\right.
$$

By Eqs. (D1) and (D2),

$$
\langle M(\alpha)|\hat{U}(\beta)| M(0)\rangle^{*}=\langle M(0)|\hat{U}(2 \pi-\beta)| M(\alpha)\rangle,
$$

where the 1-fold rotational symmetry (least rotational symmetry), unitary property of $\hat{U}$, and Eq. (A5) are used. The product of Eq. (A3) and complex conjugate of Eq. (D1) is

$$
\begin{aligned}
& \langle M(0) \mid M(\alpha)\rangle\langle M(0)|\hat{U}(\beta)| M(\alpha)\rangle^{*} \\
& =\frac{1}{4 \pi^{2}} \times\left\{\begin{array}{l}
{[\alpha(\alpha+\beta)+(2 \pi-\alpha)(2 \pi-\alpha-\beta)] e^{i \beta M}+(2 \pi-\alpha)(\alpha+\beta) e^{-i(2 \pi-\beta) M}} \\
+\alpha(2 \pi-\alpha-\beta) e^{i(2 \pi+\beta) M}, \alpha+\beta \leq 2 \pi \\
{[\alpha(\alpha+\beta-2 \pi)+(2 \pi-\alpha)(4 \pi-\alpha-\beta)] e^{-i(2 \pi-\beta) M}+(2 \pi-\alpha)(\alpha+\beta-2 \pi) e^{-i(4 \pi-\beta) M}} \\
+\alpha(4 \pi-\alpha-\beta) e^{i \beta M}, \alpha+\beta>2 \pi
\end{array} .\right.
\end{aligned}
$$

The real part of Eq. (D4) is 


$$
\begin{aligned}
& \operatorname{Re}\left[\langle M(0) \mid M(\alpha)\rangle\langle M(0)|\hat{U}(\beta)| M(\alpha)\rangle^{*}\right] \\
& =\frac{1}{4 \pi^{2}} \times\left\{\begin{array}{l}
{\left[4\left(\alpha^{2}+\alpha \beta-2 \pi \alpha-\pi \beta\right) \sin ^{2}(\pi M)+4 \pi^{2}\right] \cos (\beta M)+2 \pi \beta \sin (2 \pi M) \sin (\beta M), \alpha+\beta \leq 2 \pi} \\
2\left(\alpha^{2}+\alpha \beta-4 \pi \alpha-\pi \beta+4 \pi^{2}\right) \cos [(2 \pi-\beta) M]+(2 \pi-\alpha)(\alpha+\beta-2 \pi) \cos [(4 \pi-\beta) M] \\
+\alpha(4 \pi-\alpha-\beta) \cos (\beta M), \alpha+\beta>2 \pi
\end{array} .\right.
\end{aligned}
$$

By Eq. (D1),

$$
\begin{aligned}
& \left\langle M(0)\left|\hat{U}\left(\beta_{1}\right)\right| M(\alpha)\right\rangle\left\langle M(0)\left|\hat{U}\left(\beta_{2}\right)\right| M(\alpha)\right\rangle^{*} \\
& =\frac{e^{-i\left(\beta_{1}-\beta_{2}\right) M}}{4 \pi^{2}} \times\left\{\begin{array}{l}
4 \sin ^{2}(\pi M)\left[\left(\alpha+\beta_{1}\right)\left(\alpha+\beta_{2}\right)-\pi\left(2 \alpha+\beta_{1}+\beta_{2}\right)\right]+4 \pi^{2} \\
+i 2 \pi\left(\beta_{1}-\beta_{2}\right) \sin (2 \pi M), \alpha+\beta_{1} \leq 2 \pi \text { and } \alpha+\beta_{2} \leq 2 \pi \\
{\left[\left(\alpha+\beta_{1}\right)\left(\alpha+\beta_{2}-2 \pi\right)+\left(2 \pi-\alpha-\beta_{1}\right)\left(4 \pi-\alpha-\beta_{2}\right)\right] e^{-i 2 \pi M}} \\
+\left(2 \pi-\alpha-\beta_{1}\right)\left(\alpha+\beta_{2}-2 \pi\right) e^{-i 4 \pi M}+\left(\alpha+\beta_{1}\right)\left(4 \pi-\alpha-\beta_{2}\right) \\
\alpha+\beta_{1} \leq 2 \pi \text { and } \alpha+\beta_{2}>2 \pi \\
{\left[\left(\alpha+\beta_{1}-2 \pi\right)\left(\alpha+\beta_{2}\right)+\left(4 \pi-\alpha-\beta_{1}\right)\left(2 \pi-\alpha-\beta_{2}\right)\right] e^{i 2 \pi M}} \\
+\left(\alpha+\beta_{1}-2 \pi\right)\left(2 \pi-\alpha-\beta_{2}\right) e^{i 4 \pi M}+\left(4 \pi-\alpha-\beta_{1}\right)\left(\alpha+\beta_{2}\right) \\
\alpha+\beta_{1}>2 \pi \text { and } \alpha+\beta_{2} \leq 2 \pi \\
4 \sin ^{2}(\pi M)\left[\left(\alpha+\beta_{1}\right)\left(\alpha+\beta_{2}\right)-\pi\left(6 \alpha+3 \beta_{1}+3 \beta_{2}-8 \pi\right)\right]+4 \pi^{2} \\
+i 2 \pi\left(\beta_{1}-\beta_{2}\right) \sin (2 \pi M), \alpha+\beta_{1}>2 \pi \text { and } \alpha+\beta_{2}>2 \pi
\end{array}\right.
\end{aligned}
$$

There are four expressions in four sections of Eq. (D6). As $\beta_{1}=\beta_{2}=\beta$, Eq. (D6) becomes

$$
|\langle M(0)|\hat{U}(\beta)| M(\alpha)\rangle|^{2}=\frac{1}{4 \pi^{2}} \times\left\{\begin{array}{l}
4(\alpha+\beta)(\alpha+\beta-2 \pi) \sin ^{2}(\pi M)+4 \pi^{2}, \alpha+\beta \leq 2 \pi \\
4(\alpha+\beta-4 \pi)(\alpha+\beta-2 \pi) \sin ^{2}(\pi M)+4 \pi^{2}, \alpha+\beta>2 \pi
\end{array} .\right.
$$

The real part of Eq. (D6) is 


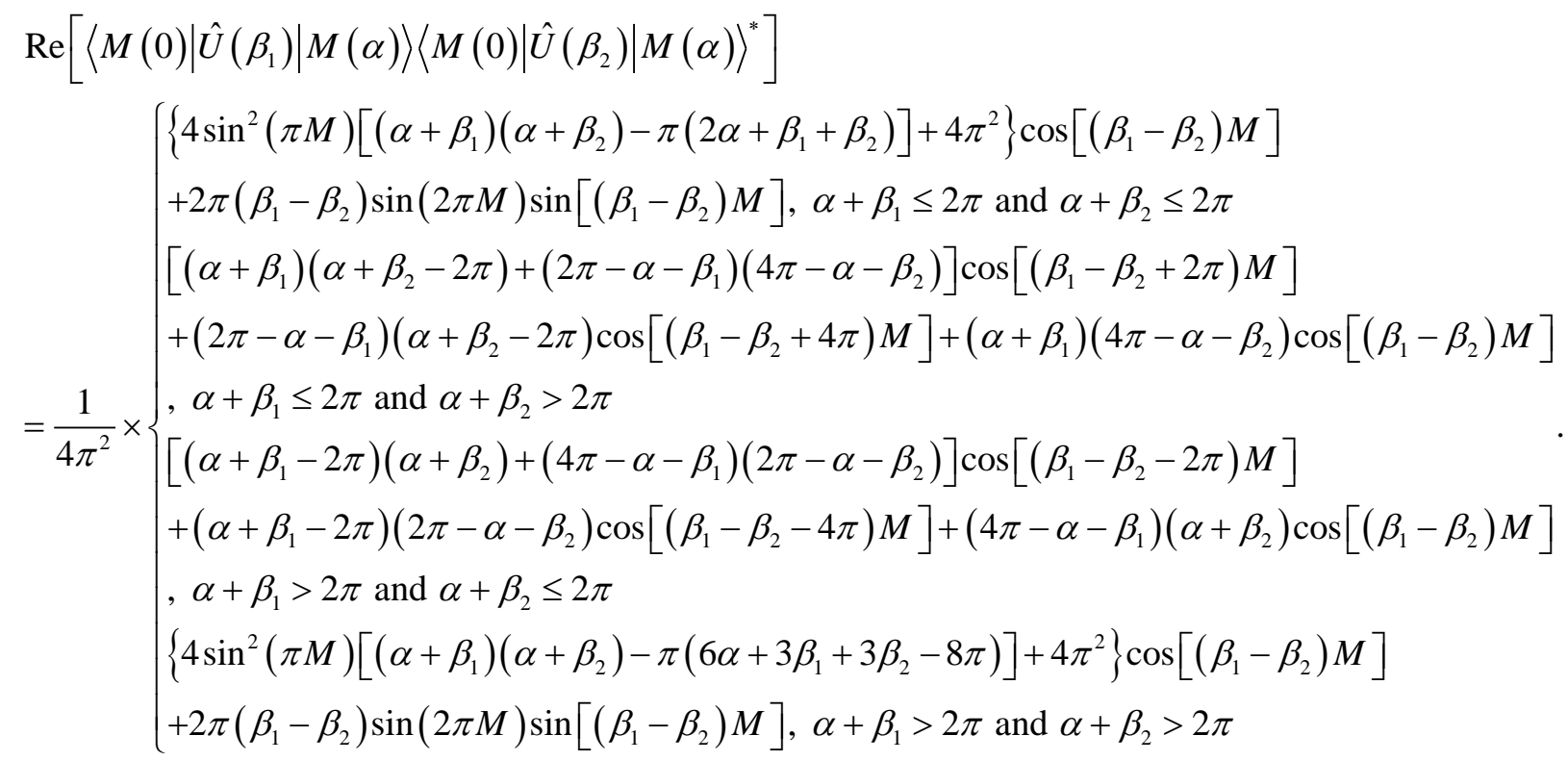




\section{References}

[1] D. N. Klyshko, A. N. Penin, and B. F. Polkovnikov, JETP lett. 11, 05 (1970)

[2] D. C. Burnham, and D. L. Weinberg, Physical Review Letters 25, 84 (1970)

[3] J. Bell, Physics 1, 195 (1964)

[4] J. F. Clauser, M. A. Horne, A. Shimony, and R. A. Holt, Physical Review Letters 23, 880 (1970)

[5] P. G. Kwiat, K. Mattle, H. Weinfurter, A. Zeilinger, A. V. Sergienko, and Y. H. Shih, Physical Review Letters 75, 4337 (1995)

[6] T. Yarnall, A. F. Abouraddy, B. E. A. Saleh, and M. C. Teich, Physical Review Letters 99, 170408 (2007)

[7] B. Jack, J. Leach, J. Romero, S. Franke-Arnold, M. Ritsch-Marte, S. M. Barnett, and M. J. Padgett, Physical Review Letters 103, 083602 (2009)

[8] J. Leach, B. Jack, J. Romero, M. Ritsch-Marte, R. W. Boyd, A. K. Jha, S. M. Barnett, S. Franke-Arnold, and M. J. Padgett, Opt. Express 17, 8287 (2009)

[9] R. Fickler, R. Lapkiewicz, W. N. Plick, M. Krenn, C. Schaeff, S. Ramelow, and A. Zeilinger, Science 338, 640 (2012)

[10] M. Padgett, J. Courtial, and L. Allen, Phys. Today 57, 35 (2004)

[11] A. M. Yao, and M. J. Padgett, Adv. in Opt. and Photon. 3, 161 (2011)

[12] A. Mair, A. Vaziri, G. Weihs, and A. Zeilinger, Nature 412, 313 (2001)

[13] H. D. Pires, H. C. B. Florijn, and M. P. van Exter, Physical Review Letters 104, 020505 (2010)

[14] H.-C. Huang, 1804.09437, (2018)

[15] D. Collins, N. Gisin, N. Linden, S. Massar, and S. Popescu, Physical Review Letters 88, 040404 (2002)

[16] A. C. Dada, J. Leach, G. S. Buller, M. J. Padgett, and E. Andersson, Nature Phys. 7, 667 (2011)

[17] J. Leach, M. R. Dennis, J. Courtial, and M. J. Padgett, New Journal of Physics 7, 55 (2005)

[18] S. S. R. Oemrawsingh, A. Aiello, E. R. Eliel, G. Nienhuis, and J. P. Woerdman, Physical Review Letters 92, 217901 (2004)

[19] S. S. R. Oemrawsingh, X. Ma, D. Voigt, A. Aiello, E. R. Eliel, G. W. 't Hooft, and J. P. Woerdman, Physical Review Letters 95, 240501 (2005)

[20] M. V. Berry, J. Opt. A: Pure. Appl. Opt. 6, 259 (2004)

[21] J. B. Gotte, S. Franke-Arnold, R. Zambrini, and S. M. Barnett, J. Mod. Opt. 54, 1723 (2007)

[22] S. N. Khonina, A. P. Porfirev, and A. V. Ustinov, Journal of Optics 17, 125607 (2015)

[23] L. Allen, M. W. Beijersbergen, R. J. C. Spreeuw, and J. P. Woerdman, Phys. Rev. A 45, 8185 (1992) 
[24] J. Leach, M. J. Padgett, S. M. Barnett, S. Franke-Arnold, and J. Courtial, Physical Review Letters 88, 257901 (2002)

[25] H. H. Arnaut, and G. A. Barbosa, Physical Review Letters 85, 286 (2000)

[26] E. R. Eliel, S. M. Dutra, G. Nienhuis, and J. P.Woerdman, Physical Review Letters 86, $5208(2001)$

[27] H. H. Arnaut, and G. A. Barbosa, Physical Review Letters 86, 5209 (2001)

[28] S. Franke-Arnold, S. M. Barnett, M. J. Padgett, and L. Allen, Phys. Rev. A 65, 033823 (2002)

[29] A. Garuccio, and V. A. Rapisarda, Nuovo Cimento Soc. Ital. Fis. 65A, 269 (1981)

[30] J. F. Clauser, and M. A. Horne, Phys. Rev. D 10, 526 (1974) 